\title{
The role of attention in different visual-search tasks
}

\author{
MARY J. BRAVO and KEN NAKAYAMA \\ Smith-Kettlewell Eye Research Institute, San Francisco, California
}

\begin{abstract}
Observers viewed displays containing a variable number of distractors of one color and a target of another color. In some experiments, the target and distractors maintained their color from trial to trial; in others, they reversed unpredictably. Observers made a speeded two-choice judg. ment concerning either the presence, the color, or the shape of the odd-colored target. With only one exception, all of these conditions produced the same pattern of results: reaction times remained constant as the number of distractors increased. The exceptional result occurred when observers judged the shape of the odd-colored target and the color of the target and distractors reversed unpredictably. In this case, reaction times decreased as the number of distractors increased. These results are interpreted in terms of the attentional requirements of the different judgments and the mechanisms that guide attention.
\end{abstract}

Figure 1 shows an odd target located among homogenous distractors. Two observations have been made concerning such displays. First, the odd element "pops out," that is, it immediately attracts attention. And second, the target is readily detected regardless of the number of distractors in the display (Donderi \& Zelnicker, 1969; Egeth, Jonides, \& Wall, 1972; Neisser, 1963; Treisman $\&$ Gelade, 1980). It has been assumed that these two observations are causally related: the target is easy to detect because it immediately summons attention (Duncan \& Humphreys, 1989; Koch \& Ullman, 1985; Yantis \& Jonides, 1984).

If we compare the predictions of models of attentional guidance with the results of detection experiments, this relationship is less clear. As detailed below, these models predict that under some circumstances the target should become easier to find as more distractors are added to the display. However, the results of detection experiments do not support this prediction. When the target and distractors have very different features, as in Figure 1, detection times are found to be unaffected by increasing numbers of distractors. When the target and distractors have similar features, detection times are found to increase with increasing numbers of distractors (Duncan \& Humphreys, 1989). Thus, the slope of detection times plotted against number of distractors is usually either zero or positive, but rarely negative (for an exception, see Bacon \& Egeth, 1991). Since the models predict a negative slope, these empirical results indicate that either the

This work was supported by Grant IF32 EY06155 from the NEI and Grant 83-0320 from the AFOSR. We are indebted to Jeremy Wolfe and Vera Maljkovic for their comments on an earlier version of this manuscript. K. Nakayama is now in the Department of Psychology at Harvard University. Correspondence should be addressed to M. J. Bravo, SmithKettlewell Eye Research Institute, 2232 Webster St., San Francisco, CA 94115; e-mail: mary@skivs.ski.org. models are incorrect or that they do not apply to target detection.

Our hypothesis is that the models of attentional guidance are correct, but that they do not apply to detection. In other words, detection does not require attention to be focused on the target. Of course, some decisions do require selective attention to be directed to the target, and it is these tasks that should show the pattern of reaction time data predicted by the models of attentional guidance. We tested this conjecture by comparing the predictions of these models with the pattern of search times for various tasks performed with identical displays. Our expectation was that these different tasks would produce different reaction time results and that only tasks requiring focal attention would produce the results predicted by the models. Before describing our experiments, we will discuss some models of how an odd target attracts attention.

\section{ATTENTION-GUIDING MECHANISMS}

There are two general ways in which attention may be directed to an odd target (Duncan \& Humphreys, 1989; LaBerge \& Brown, 1989; Wolfe, Cave, \& Franzel, 1989). When observers are looking for a particular target (say, a red diamond), they can use their knowledge of the target's characteristics to guide attention; the control of attention has a large top-down component. When observers are looking for an odd element but do not know its characteristics, then the stimulus must play a greater role in guiding attention. In this case, the control of attention is largely bottom-up. Although we will use the terms top-down and bottom-up to label these two types of attention-guiding mechanisms, we do not mean to imply that one relies solely on cognitive information and the other solely on stimulus information. Rather, the labels refer to the relative weights of these two sources of information. Below we describe these two types of attention-guiding mechanisms. 


\section{Top-Down Mechanisms}

Treisman and Gelade (1980) suggested that the visual system represents the distribution of each perceptual feature in a functionally separate map of visual space. There are maps for vertical, green, movement to the right, etc. Drawing on this notion, LaBerge and Brown (1989) proposed that if an observer knows the target's unique feature, then he/she could use the appropriate feature map to guide attention. For example, if the target is known to be the only red element in the display, then the locus of activity among red detectors may guide attention. The activity in other feature maps is ignored. Thus, if the target and distractors are so different as to stimulate different sets of feature maps, then this strategy will work equally well regardless of the number of distractors in the display. However, this strategy requires that the observer know the target's unique feature. The following bottom-up mechanisms can operate in the absence of such knowledge.

\section{Bottom-Up Mechanisms}

Koch and Ullman (1985) suggested that local inhibition within feature maps is responsible for the conspicuity of the target and its subsequent status as the focus of attention. A similar idea has been proposed by Treisman (1988) in her revised feature-integration model of visual search. If several distractors share a feature (say, the same color), the activity they generate in the corresponding feature map will be suppressed through some form of mutual inhibition. (For a review of the physiological evidence for such inhibition see Allman, Miezen, \& McGuiness, 1985.) No such suppression will occur to the activity generated by the target's unique feature because it is not subject to inhibition generated by other elements sharing this feature. The activity at each location is then summed across maps, and attention is directed to the most active location. Since the inhibitory connections between detectors in each feature map are thought to be local, it is reasonable to suppose that this process is most effective when the distractors are close together.

An alternative bottom-up mechanism comes from Julesz's proposal that pop out is the result of processes that detect feature gradients, or differences in the features of neighboring elements (Julesz, 1986). This idea posits mechanisms that compare the features of neighboring elements and then direct attention to the location of feature gradients. Because the gradient detectors operate only over a limited region of space, this process is most effective when the physical distance between distractor and target elements is small.

Since both bottom-up mechanisms involve local computations (between target and distractor in one case and between distractors in the other), they both operate most effectively when the spacing between elements is small. Thus, when attention must be directed to an unknown target, search times should depend on the spacing between elements in the display. If display size is held constant, as in these experiments, then the average spacing will decrease as the number of distractors increases. These bottom-up models predict that for tasks that require focal attention to be directed to a target with an unknown unique feature, search times should decrease as the number of distractors increases.

We now summarize our predictions for search tasks involving a target with a unique feature. (1) Two patterns of results are predicted for tasks requiring focal attention: (a) When the target's unique feature is known, reaction times should be independent of the number of distractors. (b) When this feature is not known, reaction times should decrease with increasing numbers of distractors. (2) The models mentioned above make no predictions for tasks that require distributed attention. We suggest that detection is one of these tasks, and we know from previously reported detection experiments that whether the target is known or unknown, reaction times will be unaffected by distractor number.

\section{EXPERIMENT 1 Five Search Tasks}

This series of experiments (Experiments 1A-1E) examines the pattern of reaction times for several search tasks. So that they can be readily compared, the search experiments used the same displays and the same observers. The experiments differed only in the task the observer performed. The tasks varied in two ways. First, they differed in the two-choice judgment the observer made about the odd-colored target; the observer judged either the oddcolored target's presence, its color, or its shape. These three judgments are based on information at different spatial resolutions and so are likely to differ in their requirements for distributed versus focal attention. Second, we changed the observer's prior knowledge of the target's distinguishing feature. We accomplished this by keeping the target and distractor colors the same from trial to trial in some experiments and by reversing them unpredictably in others. These two conditions are termed consistent mapping and variable mapping, after Schneider and Shiffrin (1977). These two methods for sorting the displays might produce a variation in the strategy the observer used to search for the target. In the consistent-mapping condition, the observer could search for a particular feature, whereas in the variable-mapping condition, the observer must search for the odd element. As depicted in Table 1, the two mapping conditions combined with the three judgments produced a total of five experiments. Note that the cell corresponding to consistent mapping/color judgment is blank because the color judgment is meaningless when the observer knows beforehand the target's color.

Table 1

Five Search Tasks

\begin{tabular}{lcc} 
& \multicolumn{3}{c}{ Mapping } \\
\cline { 2 - 4 } Judgment & Variable & Consistent \\
\hline Shape & $\mathrm{a}$ & $\mathrm{b}$ \\
Color & $\mathrm{c}$ & - \\
Detection & $\mathrm{d}$ & $\mathrm{e}$ \\
\hline
\end{tabular}




\section{Method}

Observers. Three practiced ${ }^{1}$ psychophysical observers volunteered for these experiments. Only one of the observers, K.N., was aware of the purpose of the experiments.

Stimuli. The displays contained red and green elements drawn on the black background of a computer monitor. The approximate luminance of the elements was $10 \mathrm{~cd} / \mathrm{m}^{2}$, and that of the background was $0.7 \mathrm{~cd} / \mathrm{m}^{2}$. To ensure that the red and green elements were essentially equiluminant so as not to bias the detection of one or the other, we used heterochromatic flicker photometry. Before participating in the experiments, each observer viewed a $1^{\circ}$ square that alternated in color between red and green. While fixating a spot $3^{\circ}$ from the square, the observer adjusted the luminance of the red to minimize the perceived flicker of the square. The observer's equiluminance setting was then used to color the red and green elements for that observer.

The colored elements were diamonds $\left(0.8^{\circ} \times 1^{\circ}\right)$ with $0.25^{\circ}$ cut off of either the right or left comer. To ensure that these two shapes (left cut and right cut) were discriminable when presented at the eccentricities used in this experiment $\left(4.75^{\circ}-8.4^{\circ}\right)$, we presented two observers with the experimental displays and monitored their eye movements. Both observers were able to confine their eye movements to within $1^{\circ}$ of a central fixation spot and at the same time perform the shape discrimination with high accuracy (>95\%).

The colored elements were drawn in randomly selected cells of a $6 \times 8$ array $\left(15.2^{\circ} \times 12.6^{\circ}\right)$. The position of the elements within the cells was jittered by $0.33^{\circ}$. Each display contained $3,5,9,13$, 25 , or 48 elements, and all but one (the target) had the same color. The position of the target was chosen randomly with the restriction that it not occur in the two columns closest to fixation. Figure 1 shows schematic representations of two such displays. The detection-task experiments required generating additional displays in which the target was replaced by a distractor on $50 \%$ of the trials.

In all five experiments, the number of distractors was varied from trial to trial. Display size was held constant, so this variation in the number of distractors also resulted in a variation in the average distance between elements in the display.

Procedure. The same procedure was used in the five search experiments, with the only differences among experiments being (1) the rule for mapping red and green onto the target and distractors and (2) the two-alternative forced-choice judgment the observer was to make. The procedural similarities are described first.

In all five experiments, the observers viewed the computergenerated displays from a distance of $70 \mathrm{~cm}$. A small fixation cross was always visible at the center of the display screen, and the observers were asked to maintain fixation throughout each experiment. Stimuli were presented with a variable interstimulus interval (ISI), and the observers responded via a two-button mouse. The display was presented until the observer responded. When the observer was correct, the reaction time was recorded. When the observer was incorrect, feedback was given and an error was recorded. Reaction times on incorrect trials were discarded, and the trials were

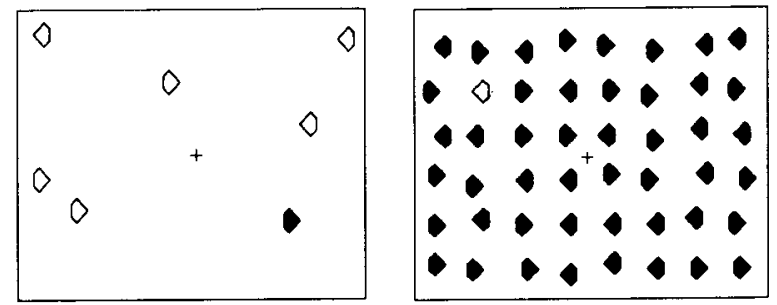

Figure 1. Schematics of representative stimuli for two distractor densities. Actual stimuli consisted of red and green shapes drawn on a black background. repeated later in the session. Reaction times less than $\mathbf{2 0 0}$ msec or greater than 2 sec were also discarded, and such trials were repeated later. Each experiment included an initial practice period involving typically 240 trials. An experimem consisted of 480 correct trials ( 80 per level of distractor number) and was run in blocks of 80 trials, with 4 practice trials preceding each block.

The experiments differed in the following ways. Within an experiment, the colors red and green were assigned to the target and distractors with either variable or consistent mapping. In the variablemapping condition, displays with a red target and green distractors were randomly interleaved among displays with a green target and red distractors. Thus, on any given trial, the observer did not know beforehand the color of the target or the distractors. In the consistentmapping condition, these two display types were run in separate blocks so that before each trial the observer knew the color of the target and distractors. In addition to the different mapping conditions, the experiments differed in the speeded forced-choice decision the observer was asked to make. The decision was always between two alternatives: either (1) present or absent in the detection experiment, (2) red or green in the color-identification experiment, or (3) left cut or right cut in the shape-identification experiment. The observers pressed the left mouse button to signal "present," "red," or "left cut" and the right mouse button to register "absent," "green," or "right cut."

\section{Results}

The results for each of the three observers in the five experiments are shown in Figure 2. Results from consistentmapping experiments are plotted with open circles; results from variable-mapping experiments are plotted with filled circles. The top two curves show the data from the shapeidentification task, the middle curve shows the data from the color-identification task, and the bottom two curves show the data from the target-detection task when the target was present. We include the data from each subject to show the remarkable consistency of the results across observers. The pertinent observation concerns the shape of these curves. Reaction times decrease as the number of distractors increases for the shape-identification, variable-mapping condition (top curve), and reaction times remain constant for all other tasks. Figure 3 (left) shows the average for the three observers.

Figure 3 (middle) shows the averaged results for the target-detection task when the target was absent. These data are plotted in a separate graph because search for an absent target is logically very different from search for a present target.

Figure 3 (right) shows the average error rates for the three observers. To determine whether a speed-accuracy tradeoff is responsible for the decrease in reaction time seen in the topmost curve in Figure 3 (left), we examined the correlation between error rates and reaction times for this condition. No correlation was found, which indicates that the observed reaction-time effect is not due to observers trading speed for accuracy as the number of distractors changed.

\section{Discussion}

Our main finding is that when observers are asked to judge the shape of the odd-colored target and the colors of the target and distractors reverse unpredictably, reaction times decrease as the number of distractors increases. 

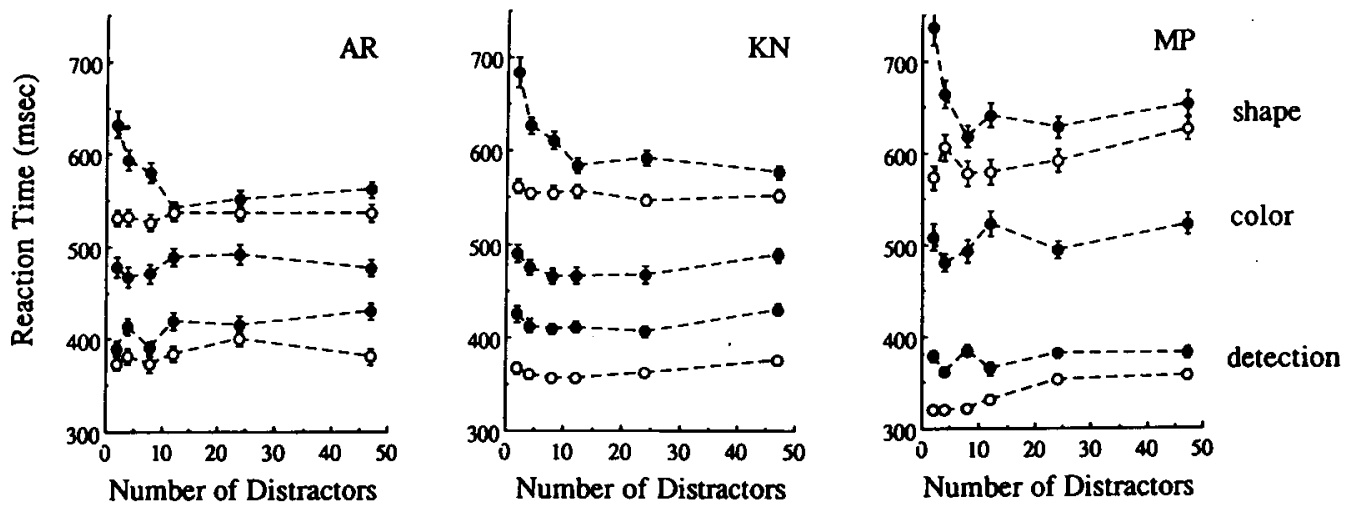

Figure 2. Average reaction time data for each of the three observers in the five different tasks. In each graph, the top two curves show data from the shape-identification judgment, the middle curve shows data from the color-identification judgment, and the bottom two curves show data from the target-detection judgment. Open circles correspond to consistent-mapping experiments and filled circles to variable-mapping experiments. Error bars show one standard error.

This effect is found only when the task involves the variable-mapping condition and the shape-discrimination judgment. What is unique about the variable-mapping condition of the shape judgment? We will argue first, that the spatial resolution required to perform the shape judgment requires focal attention, and second, that the unpredictable changes in the target's unique feature necessitate a largely bottom-up guidance of attention.

There is considerable empirical evidence suggesting that selective attention may trade off resolution and spatial extent. That is, it is possible to attend to a large visual area with low resolution or a small visual area with fine resolution (Bergen \& Julesz, 1983; Eriksen \& St. James, 1986). The necessity for this tradeoff is attributed to the limited amount of information that may be selected by attention for higher level processes like pattern recognition. We assume that different judgments require different amounts of spatial resolution. Consider the detection judg- ment under the variable-mapping condition, where the target and distractor colors are reversing unpredictably. Here, the target is defined in relation to the other elements in the display: the target is odd because it is different from the other elements in the display, which are all the same. Simply noting the presence of an odd target could be done without focusing attention on the target. In fact, focusing attention on the target gives no additional information for this task. Similarly, the color-identification judgment could logically be accomplished without directing attention to the target. For example, if observers base this decision on a comparison of the number of occurrences of the different colors, there is no obvious benefit in focusing attention on the target. However, in the shape-discrimination experiment, the observer must determine which element is the target and report about a second feature of that element. In this case, there would be a benefit in focusing attention on the target.

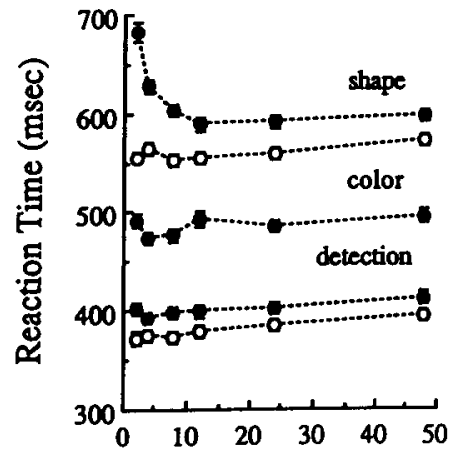

Number of Distractors

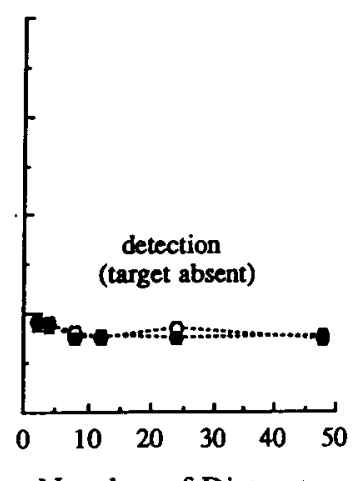

Number of Distractors

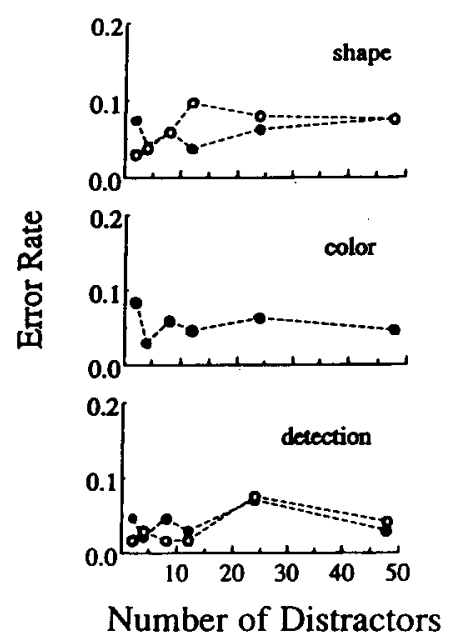

Figure 3. Left: Reaction time data shown in Figure 2, averaged across observers. Open circles correspond to consistent-mapping experiments and filled circles to variable-mapping experiments. Error bars show one standard error. Middle: Average reaction time for the detection task when the target was absent from the display. Right: Average error rates for the three observers. 
But the effect cannot simply be ascribed to the involvement of a small attentional aperture in shape discrimination since the effect did not appear under a different condition involving this judgment. When the shape-discrimination judgment was made under the consistent-mapping condition, reaction times were unaffected by the number of distractors. Thus, the other critical requirement for the distractor-number effect is that the observer not know the target's color before the trial. We believe that the significance of this requirement relates to the two classes of attention-guiding mechanisms discussed in the introduction. Recall that top-down guidance can direct attention to the target when the target's unique feature is known, and that the effectiveness of this mechanism is expected to be independent of the number of distractors. Bottomup guidance is required when the target's unique feature is not known, and this mechanism depends on the number of distractors.

Thus, we claim the variable-mapping/shape-judgment task is unique because it requires an attention-guiding mechanism that is driven by largely bottom-up information. However, the shape judgment may have also been unique in that the relatively high resolution required to make this judgment may have caused the subjects to make eye movements. Since we did not monitor fixation during these search tasks, it is possible that our observers made eye movements during the shape-judgment experiment but not during the other experiments. Thus, it could be argued that the different reaction-time pattern obtained for these judgments is related to the occurrence of eye movements for the shape judgments and the absence of eye movements for the other judgments.

Would this alter our interpretation? We think not. If we assume for purposes of argument that the subjects made saccades to the target location only when asked to judge the shape of the target, we need to consider if this would change our explanation of the distractor-number effect.

First, we know that the observed effect of distractor number cannot simply be due to the putative eye movement itself, since there was no such effect when the subject made a saccade to a target whose distinguishing feature was known. The effect must instead be related to directing a saccade to an unknown target. Previously, we had assumed that because the shape-discrimination task requires relatively high resolution, it involves a shift in focal attention to the target. If we replace this assumption with the assumption that saccades involve a shift in focal attention to the target, then we are left with our original conclusion: tasks that require focal attention give the pattern of results predicted by the attention-guiding mechanisms. Our new assumption, that saccades are preceded by a shift in focal attention, is commonly made and has some empirical support (Fischer \& Breitmeyer, 1987; Groner \& Groner, 1989; Mayfrank, Mobashery, Kimmig, \& Fischer, 1986; Posner, 1980; Remington, 1980). For the remainder of this paper, we will assume that the observers were not making eye movements, but our interpretation of these experiments would not change substantially if we were to assume the opposite.

\section{EXPERIMENT 2 \\ Replication of Set-Size Effect With Gabor-Patch Stimuli}

The data from the first set of experiments show that the effect of distractors on visual search may depend on the observer's task. This suggests that "finding" a feature target in a detection experiment is different from "finding" a feature target in order to identify a subtle feature. We have argued that this difference has to do with whether the task requires focal attention to be directed to the target. Our results also suggest that different attention-guiding mechanisms may be invoked depending on the observer's prior knowledge of the target's characteristics. To examine the generality of our results, we repeated the variable-mapping and consistent-mapping experiments using different stimuli. Observers were again asked to make a fine spatial judgment concerning a nonunique feature of the odd element.

\section{Method}

We generated new stimuli consisting of Gabor patches ${ }^{2}$ of either high or low spatial frequency and with a vermier offset to the left or right. Spatial frequency replaced color as the unique feature, and the direction of a vernier offset replaced shape as the second, nonunique feature of the target. Observers were required to determine whether the vernier offset was to the right or left in the patch with the odd spatial frequency. Figure 4 shows schematic representations of two of these displays. The procedure was the same as in Experiment 1, and the details of the stimuli are given below.

The target and distractor elements were vertically oriented Gabor patches with spatial frequency centered on 3 cycles per degree or on 1 cycle per degree. Each patch had a vernier offset $4 \mathrm{~min}$ to the left or right. These patches were drawn in the cells of a $4 \times 3$ array $\left(15.2^{\circ} \times 12.6^{\circ}\right)$ with a jitter of $0.66^{\circ}$. Displays contained 2, $3,4,6$, or 12 elements.

Two observers participated in this experiment, one of whom had been involved in the previous experiment. The other observer, R.O., was paid to participate. R.O. did not know the purpose of the experiment and had never before acted as an experimental observer.

\section{Results and Discussion}

The results of the two observers are given in Figure 5. Although the two observers have very different reaction times, possibly due to different levels of experience in psychophysical tests, both show the same pattern of results. As with the previous experiment, reaction times decrease as the number of distractors increases only when the distinguishing features of the target and distractor are

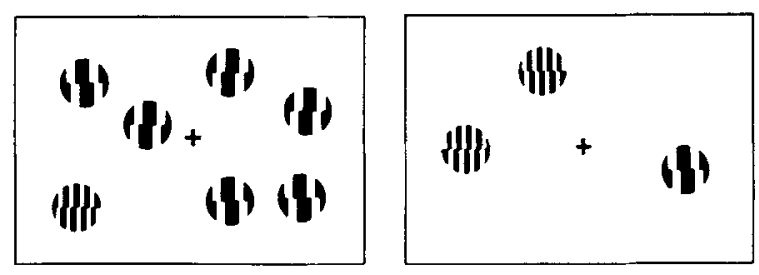

Figure 4. Schematic of two stimuli composed of Gabor functions. In the actual experiment, luminance varied more smoothly as it was the product of a sinusoid and a Gaussian waveform. 

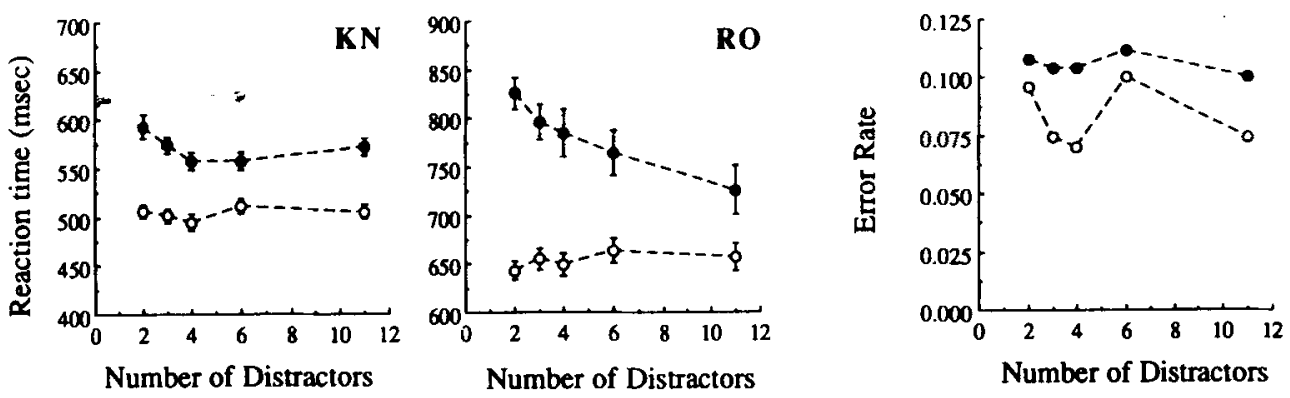

Figure 5. Left, middle: Reaction time to judge the direction of the vernier offset of the odd-spatial-frequency target plotted against the number of distractors in the display. Note the different values on the $y$-axes of these graphs. Open circles represent data from the consistent-mapping experiment; filled circles represent data from the variablemapping experiment. Error bars show one standard error. Right: Average error rates for the three observers.

interchanged unpredictably from trial to trial. And again, this effect cannot be ascribed to a speed-accuracy tradeoff since there is no significant correlation between error rate and reaction time.

This second pair of experiments employed very different stimuli than did the previous experiments, and yet the same overall pattern of results was obtained. When the unique feature of the target was consistent across trials, reaction times to identify a subtle feature of the target were unaffected by the number of distractors. When the unique feature of the target reversed unpredictably with the distractor feature, reaction times decreased as the number of distractors increased.

\section{GENERAL DISCUSSION}

The pattern of our reaction time results reveals fundamental differences in the processes invoked by different visual-search tasks. One difference can be seen in the results of the three experiments involving different judgments under the variable-mapping condition. Reaction times for the discrimination of a subtle feature of the target decrease as the number of distractors increases, while reaction times to detect the target or identify its unique feature are unaffected by the number of distractors. This discrepancy, we believe, is due to the involvement of focal attention in the first case and distributed attention in the others. A second difference among the processes involved in different visual-search tasks can be seen in the results of the two experiments involving shape identification under different mapping conditions. If we assume that the shape judgment requires focal attention, then these results suggest the existence of two classes of mechanisms by which focal attention can be directed to the target. These are (1) a largely top-down mechanism that requires knowledge of the target and (2) a largely bottom-up mechanism that can operate without such knowledge. As discussed earlier, current models of bottom-up mechanisms predict our finding that reaction times fall off sharply as the number of distractors increases. The models do not predict the results of the detection- and unique-feature- identification tasks, and in the following paragraphs, we consider the processes that might be involved in these tasks.

We consider first the task of determining the color of the odd-colored target. There are several strategies that an observer could use to judge the color of the target. One strategy is to determine which color has the smaller number of occurrences in the display. Increasing the number of distractors in the display would increase the difference in the number of occurrences of the two colors and presumably facilitate this decision. A similar strategy is to determine which color has more than one occurrence in the display and then to choose the other color. Again, it is reasonable to expect that increasing the number of distractors in the display would facilitate this decision. However, we found that the reaction times to judge the color of the target were unaffected by the number or distractors.

It is also not clear how observers detected the presence of an odd target in the detection experiments. Processes similar to the attention-guiding mechanisms outlined in the introduction have been suggested. In the consistentmapping/detection experiment, the observer could respond on the basis of the presence or absence of activity in the appropriate feature map (Treisman \& Gelade, 1980). And in the variable-mapping/detection experiment, observers could use one of the bottom-up models to detect the presence of an odd element (Julesz, 1986; Koch \& Ullman, 1985). But if the computations involved in these bottomup mechanisms are local, then search times should be sensitive to the number of distractors in the display. Although there are numerous reports that the number of distractors has no effect on searching for a readily segregated target, Bacon and Egeth (1991) recently reported that detection times may decrease as the number of distractors increases. In our experiments, however, the same conditions that produced a large effect for the shape judgment produced no effect for the detection judgment. Thus, our observers are not using the same processes to find the target for detection and shape identification. It also appears that the process they use for detection is insensitive to the number of distractors. 
We favor an alternative explanation of the detection and unique-feature-identification results that relates to coarsescale pattern matching. We assume that each of our tasks involves the postattentive processes of pattern recognition but that the particular pattern that is to be recognized depends on the observer's judgment. Sometimes the pattern is that of an individual element, as in the shape judgment; other times it is that of the whole display, as in the detection and unique-feature-identification judgment. Because it is the attentional window that selects the information for pattern recognition, these different tasks require attention to operate in different ways. It is these different attentional operations that are reflected in the reaction time results. When the display as a whole is used for pattern recognition, reaction time is unaffected by the number of distractors in the display. When only a part of the display is used, then attention must be directed to the appropriate part.

There are several ways attention may be directed, and each is associated with a characteristic pattern of reaction time results. We have already discussed top-down mechanisms, which guide attention to a particular feature (flat-search function), and bottom-up mechanisms, which guide attention to an odd target (negative-search function). There is a third type of attentional guidance that we did not see in these experiments but that has been reported by many others. A serial search through all elements in the display is required if discriminating between the target and distractor requires focal attention. This type of search produces positive search functions (Estes \& Wessel, 1966; Treisman \& Gelade, 1980).
In closing, we refer the reader to a general framework of vision proposed by Nakayama (1990) that has greatly influenced our thinking about these experiments. The model has three components, as depicted in Figure 6. On the left of this figure, incoming sensory information is encoded by a massively parallel system that represents multiple features at multiple scales. This system has a pyramid-like structure, with each level in the pyramid representing the visual field at a different spatial scale. The base of the pyramid is a very fine scale representation, and the apex is a very coarse representation. The amount of information contained at any level is directly related to the resolution of that level.

On the right in Figure 6 is visual memory, an associative network of icons or templates. Pattern recognition is accomplished by activating an icon in visual memory. It is assumed that individual icons have a low information content. This assumption is based in part on the results of reading studies that assess the amount of information that is processed simultaneously (Legge, Pelli, Rubin, \& Schleske, 1985; Rayner, 1978).

In the middle in Figure 6 is selective attention, the link between the massive amount of information in the pyramid and the small amount of information in an icon. Attention can be thought of as an information bottleneck through which only a small set amount of information may pass. Attention-guiding mechanisms are required to select the appropriate information from the pyramid to be passed on to visual memory.

This model offers an explanation of how the tradeoff between spatial extent and resolution is accomplished prior

\section{Pyramid Attention Visual Memory}

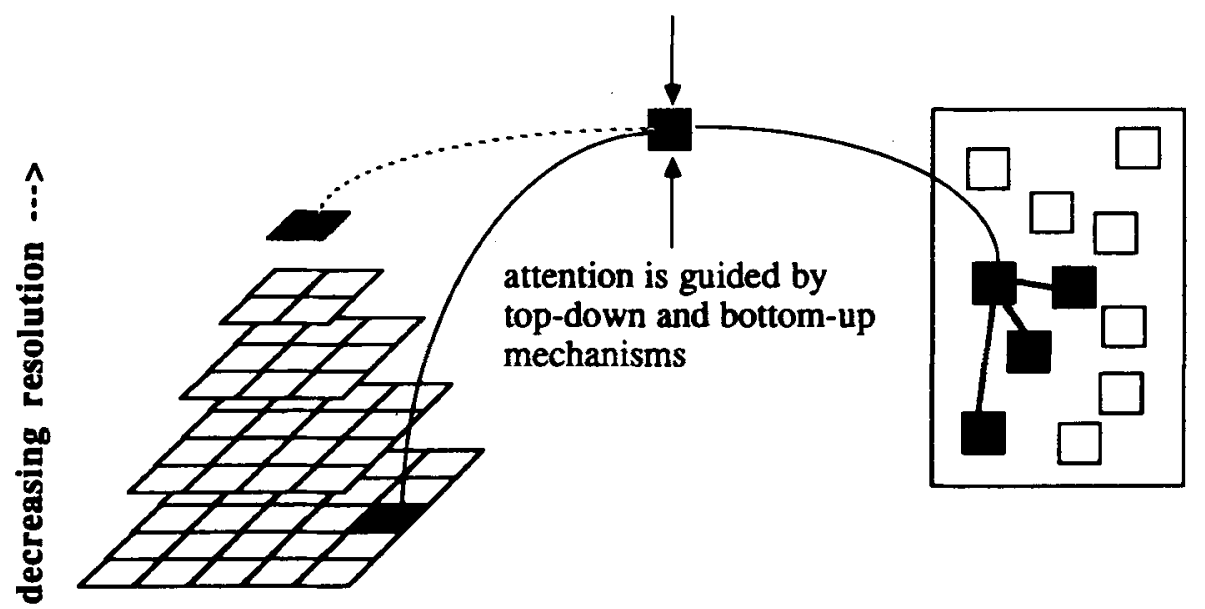

Figure 6. A schematic of the model proposed by Nakayama (1990) applied to the present results. Sensory information is encoded in a multiresolution pyramid. Attention selects the information from the pyramid that is to be used for pattern matching. The continuous line depicts the selection of information for the shapediscrimination judgment. Attention-guiding mechanisms are required to direct attention to the appropriate location within a high-resolution level of the pyramid. The hatched line indicates the selection of information for the detection judgment. 
to pattern recognition. Depending on the observer's task, detailed information about a small spatial region may be selected from a level close to the base of the pyramid or, alternatively, information about the coarsest scene features may be selected from a level close to the apex. In our experiments, observers knew before every trial the judgment, and thus the resolution, that would be required. This allowed them to select the appropriate level within the pyramid before each trial. However, they did not know the location of the target within the stimulus, and thus when high resolution was required, they had to draw on attention-guiding mechanisms to select the appropriate location within the high-resolution level of the pyramid. It is this latter step that is reflected in the unusual reaction time results reported here.

\section{REFERENCES}

Allman, J., Miezen, F., McGuiness, E. (1985). Stimulus specific responses from beyond the classical receptive field: Neurophysiological mechanisms for local-global comparisons in visual neurons. Annual Review of Neuroscience, 8, 407-430.

BACON, W., EGETH, H. (1991). Local processes in preattentive feature detection. Journal of Experimental Psychology: Human Perception \& Performance, 17, 77-90.

BERGEN, J., JULEsz, B. (1983). Rapid discrimination of visual patterns. I.E.E.E. Transactions on Systems, Man \& Cybernetics, SMC-13, 857-863.

DONDER, D. C., \& ZelniCker, D. (1969). Parallel processing in visual same-different decisions. Perception \& Psychophysics, 5, 197-200

Duncan, J., \& Humphreys, G. W. (1989). Visual search and stimulus similarity. Psychological Review, 96, 433-458.

EGETH, H., JoNIDES, J., W WALL, S. (1972). Parallel processing of multielement displays. Cognitive Psychology, 3, 674-698.

ERIKSEN, C. W., \& ST. JAmES, J. D. (1986). Visual attention within and around the field of focal attention: A zoom lens model. Perception \& Psychophysics, 40, 225-240.

Estes, W. K., \& WesseL, D. L. (1966). Reaction time in relation to display size and correctness of response in forced-choice visual signal detection. Perception \& Psychophysics, 1, 369-373.

Fischer, B., \& BreITMEYER, B. (1987). Mechanisms of visual attention revealed by saccadic eye movements. Neuropsychologia, 25 , 73-83.

Groner, R., \& Groner, M. T. (1989). Attention and eye movement control: An overview. European Archives of Psychiatry \& Neurological Sciences, 239, 9-16.

Julesz, B. (1986). Texton gradients: The texton theory revisited. Biological Cybernetics, 54, 245-251.

Kосн, C., Ullman, S. (1985). Shifts in selective visual attention: Towards the underlying neural circuitry. Human Neurobiology, 4 , 219-227.

LABerge, D., Brown, V. (1989). Theory of attentional operations in shape identification. Psychological Review, 96, 101-124.
LegGe, G., Pelli, D., Rubin, G. S., \& Schleske, M. M. (1985) Psychophysics of reading. I. Normal vision. Vision Research, 25, 239-252.

Mayfrank, L., Mobashery, M., Kimmig, H., Fischer, B. (1986). The role of fixation and visual attention in the occurrence of express saccades in man. European Archives of Psychiatry \& Neurological Sciences, 235, 269-275.

Nakayama, K. (1990). The iconic bottleneck and the tenuous link between early visual processing and perception. In C. Blakemore (Ed.), Vision: Coding and efficiency (pp. 411-422). Cambridge: Cambridge University Press.

NeISSER, U. (1963). Decision-time without reaction time: Experiments in visual scanning. American Journal of Psychology, 76, 376-385.

Posner, M. (1980). Orienting of attention. Quanerly Journal of Experimental Psychology, 32, 3-25.

RAYNER, K. (1978). Eye movements in reading and information processing. Psychology Bulletin, 85, 618-660.

REMINGTON, R. W. (1980). Attention and saccadic eye movements. Journal of Experimental Psychology: Human Perception \& Performance, 6. 726-744.

SCHNeIDER, W., \& SHIFFrun, R. M. (1977). Controlled and automatic human information processing. I. Detection, search, and attention. Psychological Review, 84, 1-66.

Treisman, A. (1988). Feature and objects: The fourteenth Bartlett memorial lecture. Quarterly Joumal of Experimental Psychology, 40A 201-237.

Treisman, A., Gelade, G. (1980). A feature-integration theory of attention. Cognitive Psychology, 12, 97-136.

Wolfe, J. M., CAve, K. R., \& Franzel, S. L. (1989). Guided search: An alternative to the feature integration model of visual search. Joumal of Experimental Psychology: Human Perception \& Performance, 15, 419-433.

YANTIS, S., JonIDES, J. (1984). Abrupt visual onsets and selective visual attention: Evidence from visual search. Joumal of Experimental Psychology: Human Perception \& Performance, 10, 601-621.

\section{NOTES}

1. By practiced observers, we mean observers who have participated in a variety of experiments and are familiar with psychophysical procedures. We have not included the observations from M.B. (the first author) because in the process of developing the test described in the present research, she had large and differing amounts of practice on each task. In addition, in using her as an observer over many trials, we found that some of the differences in reaction time described in this paper decrease with very prolonged practice.

2. Gabor patches are spatially localized sine-wave gratings, whose modulation envelope is a two-dimensional Gaussian function. The Gaussian window of the Gabor patches had a width of $0.62^{\circ}$ when the envelope's amplitude was $1 / e$, and the suprathreshold area of the Gabor patches subtended approximately $2^{\circ}$.

(Manuscript received August 19, 1991; revision accepted for publication December 27, 1991.) 